\title{
Angiogenesis modulates adipogenesis and obesity
}

\author{
Yihai Cao \\ Department of Microbiology, Tumor and Cell Biology, Karolinska Institute, Stockholm, Sweden.
}

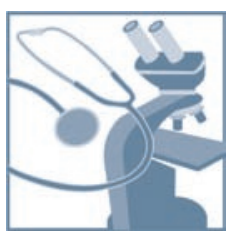

\begin{abstract}
Substantial evidence shows that neoplastic and nonneoplastic tissue growth is dependent on angiogenesis. Neovascularization and adipogenesis are temporally and spatially coupled processes during prenatal life and they continue to reciprocally interact via paracrine signaling systems throughout adult life. Activated adipocytes produce multiple angiogenic factors including leptin, angiopoietins, HGF, GM-CSF, VEGF, FGF-2, and TGF- $\beta$, which either alone or collectively stimulate neovascularization during fat mass expansion. Thus antiangiogenic agents provide a novel therapeutic option for prevention and treatment of human obesity and its related disorders.
\end{abstract}

\section{Introduction}

Approximately $65 \%$ of adults in the United States and more than half of the population in the rest of developed countries are overweight or obese (1). The prevalence in children in all developed countries is about $15 \%$ and this number is expected to increase significantly in the near future. Obesity is a complex metabolic disorder that is commonly associated with type 2 diabetes mellitus, hypertension, coronary heart disease, stroke, dyslipidemia, gallbladder disease, hepatic steatosis, sleep apnea, stroke, endometrial disorder, and cancer (2-4). Interestingly, most of these obesity-related disorders are closely associated with vascular dysfunctions. For example, hyper- or hypovascularization could result in onset and progression of diabetic ocular and kidney complications, cardiovascular disease, stroke, and cancer (5-12). Adipose tissue is highly vascularized, and each adipocyte is nourished by an extensive capillary network (13-15). Adipose tissue is considered as the largest endocrine gland because it produces free fatty acids, hormones, growth factors, and cytokines such as leptin, adiponectin, resistin, VEGF, HGF, IGF-1, angiogenin, IL-6, TNF- $\alpha$, and angiopoietins (Angs). Recently angiogenesis inhibitors have been shown to inhibit fat mass expansion in mice (16-18). These findings have paved avenues for possible therapeutic intervention of obesity and obesity-associated disorders by targeting the vascular compartment.

\section{Functional link between angiogenesis and adipogenesis}

During embryogenesis, adipose tissue development is spatially and temporally associated with microvessel growth (14). Endothelial cells isolated from different adipose tissues differ in their proliferative capacity, which suggests that adipocytes play both guidance and maintenance roles in vascular development $(19,20)$. A recent study suggests that adipocytes and their accompanying endothelial cells might share a common progenitor that could differentiate into adipocytes or endothelial lineages depending upon exposure to different environments (21). Human adipose tissue-derived stem cells can differentiate into endothelial cells and

Nonstandard abbreviations used: Ang-1, angiopoietin 1; ASC, adipose stromal cell; BAT, brown adipose tissue; CAM, chick chorioallantoic membrane; NPY, neuropeptide Y; PlGF, placental growth factor; TF, tissue factor; TSP-1, thrombospondin 1; WAT, white adipose tissue.

Conflict of interest: The author has declared that no conflict of interest exists. Citation for this article: J. Clin. Invest. 117:2362-2368 (2007). doi:10.1172/JCI32239. improve postnatal neovascularization (22). These findings raise an interesting and exciting possibility that targeting a common adipose progenitor is probably an effective approach for therapeutic intervention of obesity.

Brown adipose tissue (BAT) has a high rate of energy expenditure, but it remains functionally quiescent in obesity $(23,24)$. The high thermogenic activity of BAT requires a particularly high rate of blood perfusion to supply $\mathrm{O}_{2}$ and substrates and to export heat. Angiogenesis is essential for BAT hyperplasia, which relies on a rapid activation of mitosis in brown fat precursor cells and endothelial cells to form capillaries (25). White adipose tissue (WAT) can be converted into BAT under certain circumstances such as a chronic exposure to cold (26). This transition might be accompanied by switching on an angiogenic phenotype. Conversely, transformation of BAT into WAT might lead to regression of certain capillary networks.

Adipose tissue has been long known to promote wound healing and to revascularize ischemic tissues including myocardium $(27,28)$. These findings suggest that adipose tissue produces angiogenic molecules. Experimental angiogenesis assays show that conditioned media obtained from preadipocytes and tissue homogenates from omentum or subcutaneous fat induce angiogenesis in the chick chorioallantoic membrane (CAM) and in the mouse cornea $(15,29,30)$. BM-derived circulating endothelial precursor cells (CEPCs) do not seem to significantly contribute to adipose neovascularization although these cells are known to participate in neovascularization in other tissues $(11,17)$. For example, VEGF is a potent chemoattractant factor for inflammatory cells and for mobilization of BM-derived CEPCs, which participate in tumor neovascularization (11). Interestingly, expression levels of VEGF are only moderately upregulated in growing adipose tissue although it is a major angiogenic factor in omentum $(31,32)$.

\section{Crosstalk between endothelial cells and adipocytes}

Accumulating evidence shows that capillary endothelial cells communicate with adipocytes via paracrine signaling pathways, extracellular components, and direct cell-cell interactions (13, 33, 34). In developing embryos, the formation of primitive fat organs occurs at the perivascular site (35). Human preadipocytes and capillary endothelial cells express $\alpha_{v} \beta_{3}$ integrin and plasminogen activator inhibitor 1 , which guide preadipocyte migration toward developing capillary networks to ensure the coordination of the 


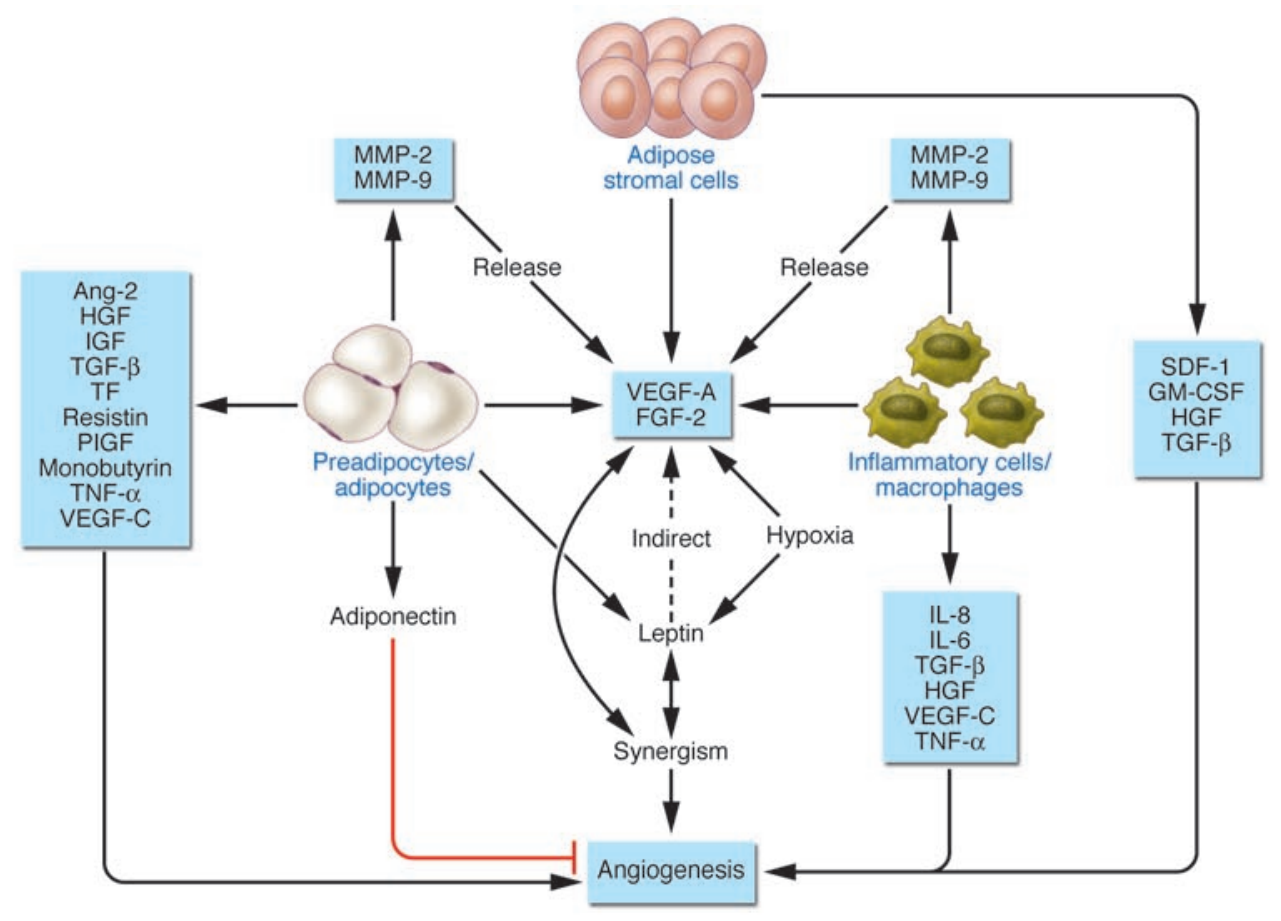

Figure 1

Regulation of adipose tissue angiogenesis by multiple factors. Diversity of cell populations in the adipose tissue, including preadipocytes and adipocytes, ASCs, and inflammatory cells contributes to production of multiple angiogenic factors and inhibitors that regulate adipose angiogenesis. Interplay between different factors could result in angiogenic synergism. For example, leptin and VEGF-A or FGF-2 could synergistically stimulate angiogenesis. Tissue hypoxia could further upregulate expression levels of VEGF and leptin. MMPs could increase bioavailability of angiogenic factors by release of the matrix-bound VEGF.

development of both tissues at the same locus (36). Further, the anatomical location of adipose depots or fat pads could also affect the pattern and function of the vasculature. PPAR- $\gamma$, as an essential mediator for preadipocyte differentiation, is involved in regulation of adipose angiogenesis (37-41). Interestingly, inhibition of adipocyte differentiation by overexpression of a dominant-negative PPAR- $\gamma$ construct leads to impaired development of both adipose tissue and angiogenesis (37). Blockade of the VEGFR-2 signaling system by a neutralizing antibody inhibits both angiogenesis and preadipocyte differentiation, suggesting that VEGF acts on endothelial cells to regulate preadipocyte differentiation (37).

Maturation of capillary networks and the size of fetal adipose clusters are inversely correlated with the degree of ECM deposition, and the presence of Matrigel reduces preadipocyte and microvessel maturation $(14,42)$. Adipose tissue produces several MMPs including MMP-2 and -9, which could potentially affect preadipocyte differentiation and microvessel maturation by modulating ECM (43). Moreover, MMP-9 is able to release the matrixbound VEGF and indirectly induces angiogenesis (44). Indeed, several lines of evidence suggest that endogenous and exogenous MMPs regulate adipogenesis (42, 43, 45). In addition to MMP-2 and MMP-9, upregulation of MMP-3, $-11,-12,-13$, and -14 and downregulation of MMP-7, $-9,-16$, and -24 have been found in expanding adipose tissues although most of these modulations are specific to gonadal fat depots (45). It is worthwhile to mention that accelerated development of high-calorie diet-induced obesity is observed in MMP-3-deficient (stromelysin-1-deficient) mice (46). Adipose blood vessel density is also increased in these knock- out mice, suggesting that MMP-3 modulates adipose angiogenesis (46). Deletion of tissue inhibitor-1 of MMP (TIMP-1), a known angiogenesis inhibitor, leads to reduced obesity in mice fed a highfat diet (47). In contrast, significantly higher vessel density and sizes are present in the adipose tissue of TIMP-1 knockout mice compared with control mice. These paradoxical findings might be explained by normalizing blood vessel density with the number of adipocytes $(45,47)$. Treatment of high fat diet-fed mice with galardin, a broad-spectrum inhibitor of MMP and angiogenesis, leads to significant reduction of fat deposits, although blood vessel density is relatively increased due to decreased size and number of adipocytes (48). Collectively, these findings demonstrate that MMPs and TIMPs play a pivotal role in controlling adipogenesis via regulation of angiogenesis.

In rapidly expanding adipose tissue, hypoxia is another important factor for vascular growth and remodeling (13). In response to hypoxia, adipose tissues produce hypoxia inducible factor 1ainduced angiogenic factors such as VEGF, leptin, TNF- $\alpha$, and PAI-1, which regulate angiogenesis and vasculogenesis $(49,50)$. Thus it is reasonable to speculate that expansion of adipose tissue is associated with local hypoxia, which contributes to angiogenesis by induction of a number of growth factors. In agreement with this hypothesis, a recent study showed that the tip region of adult epididymal adipose is extremely hypoxic and expresses high levels of VEGF, VEGF receptors, MMPs, and stromal cell-derived factor 1 , which collectively attract accumulation of BM-derived lymphatic endothelium hyaluronan receptor-positive macrophages (51). In this context the macrophage-induced angiogenesis may play an important role in 


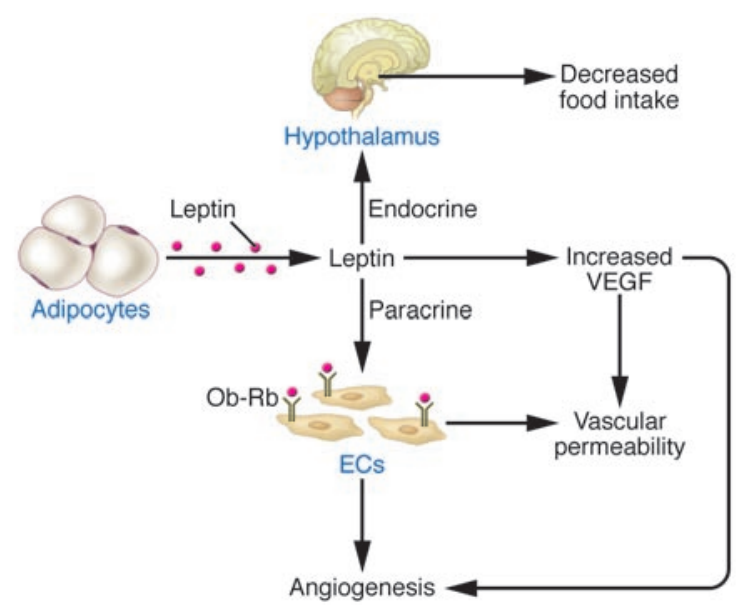

Figure 2

Role of leptin in regulation of adipose angiogenesis. Leptin directly induces angiogenesis by activation of its functional receptor, Ob-Rb, expressed on endothelial cells. In addition to its angiogenic activity, leptin is also able to induce vascular fenestrations and permeability. Leptin could indirectly induce angiogenesis via upregulation of VEGF expression.

guiding adipogenesis. Under hypoxic conditions, endothelial cells release soluble factors that maintain preadipocyte viability (48).

\section{Adipose tissue-derived angiogenic factors}

Growing adipose tissue contains a diversity of cell types including adipocytes, adipose stromal cells (ASCs), endothelial cells, and inflammatory cells (52). The diversity of heterogeneous cell populations determines expression of multiple growth factors and cytokines that either individually or jointly regulate vessel growth, although little is known about the functional interplay between these factors (53). Growing adipocytes produce a dozen angiogenic factors including leptin, VEGF, FGF-2, HGF, IGF, TNF- $\alpha$, TGF- $\beta$, placental growth factor (PlGF), VEGF-C, resistin, tissue factor (TF), neuropeptide Y (NPY), heparin-binding epidermal growth factor, and Angs (31,54-62). Preadipocytes and adipocytes also produce non-protein small lipid molecules such as monobutyrin that stimulate angiogenesis in the adipose tissue $(63,64)$. ASCs secrete high levels of a number of angiogenic factors including VEGF, HGF, GM-CSF, FGF-2, and TGF- $\beta$ (43). Recruitment of inflammatory cells also significantly contributes to adipose neovascularization. For example, activated macrophages produce potent angiogenic factors such as TNF- $\alpha$, VEGF, FGF-2, IL-1b, IL-6, and IL-8 $(65,66)$ (Figure 1).

Leptin is an adipocyte-derived hormone that regulates food intake and energy homeostasis. Functional impairment of leptin leads to severe obesity, diabetes, and infertility (55). Interestingly, leptin is also defined as a potent angiogenic factor. The initial finding that endothelial cells express the functional long form of leptin receptor $(\mathrm{OB}-\mathrm{Rb})$ has led to the discovery of its angiogenic activity (67). Interaction of leptin with its OB-Rb receptor in endothelial cells results in activation of the Stat 3 pathway and enhancement of its DNA binding activity (67). It promotes endothelial cell tube formation in vitro and corneal neovascularization in vivo $(16,67,68)$. Consistent with these findings, topical administration of leptin to wound beds accelerates wound repair in $o b / o b$ mice but not in $d b / d b$ mice (69). In addition to its direct angiogenic activity, leptin upregulates VEGF mRNA expression via activation of the Jak/Stat3 signaling pathway (70). In CAM assay, inhibition of FGF-2 function partially impairs leptin-induced neovascularization in the developing embryo, suggesting that FGF-2-triggered signaling pathways in endothelial cells are essential for leptin-induced angiogenesis (71). Consistent with this notion, leptin has been shown to induce MMP-2 and MMP-9 activity, which indirectly facilitates angiogenesis (72). Interestingly, leptin modulates FGF-2- and VEGF-induced vascular activity by synergistically promoting neovascularization in vivo (73). These studies show that leptin also acts as an indirect angiogenic factor or a modulator for other known angiogenic factors (Figure 2). Under hypoxia, the expression ratios of angiogenic factors in adipose tissue might be altered because expression levels of VEGF and leptin are elevated by low oxygen (74-76). Thus hypoxia probably plays a critical role in controlling the balance between vessel growth and remodeling by differentially regulating expression levels of various angiogenic factors.

Other vascular functions of leptin include regulation of vasodilation and vascular permeability. Leptin induces endothelial NOS activity, which increases vasodilation and blood perfusion in the adipose tissue $(77,78)$. The vasodilation function of leptin may be essential to comply with the demand of growing adipose tissues for an elevated blood flow rate. Indeed, leptin is able to increase blood perfusion in adipose tissue. Growing adipose tissue contains fenestrated capillaries that are essential for vascular permeability $(79,80)$. Similar to adipose tissue, high levels of leptin are found in other angiogenic tissues including developing placenta, mammary glands, and skeletal muscles, validating the peripheral role of leptin in vascular development $(81,82)$. NPY is probably another example of small protein peptides acting as both endocrine and paracrine factors to control adipogenesis and obesity. NPY stimulates angiogenesis in vitro and in vivo via activation of its Y2 receptor distributed in vascular endothelial cells, and deletion of the Y2 receptor in mice leads to delayed wound healing (83-85).

VEGF plays a central role in most growing or developing healthy and pathological tissues. Among all adipose tissues examined in the body, omentum expresses the highest level of VEGF (32). Localization studies have shown that adipocytes are the primary source of VEGF, which may act as an angiogenic and vascular survival factor for the omental vasculature. Additionally, adipose-infiltrated inflammatory cells and ASCs also significantly contribute to VEGF production $(51,52,86)$. Inactivation of PlGF (another member of the VEGF family) function in mice leads to impaired adipose tissue development due to defective angiogenesis, suggesting that other VEGF members also modulate adipogenesis via the vascular system (58).

Gene expression profile analysis of rat WAT shows a differential expression pattern of adipokines including leptin, adiponectin, and resistin in growing versus quiescent adipose tissues (31). Interestingly, resistin, a specific adipokine, is also defined as a novel angiogenic factor that directly promotes endothelial cell proliferation, migration, and tube formation (59). IGF- 1 and TNF- $\alpha$ are two other upregulated angiogenic factors in expanding adipose tissues (31). IGF-1 is a survival factor for many cell types and may play an important role in maintenance of vascular integrity in adipose tissue (87). In addition to its direct angiogenic activity, TNF- $\alpha$ is a potent inflammatory cytokine that links between inflammation, angiogenesis, and adipogenesis (88). In fact, adipose-infiltrated inflammatory cells produce high levels of proangiogenic cytokines such as TNF- $\alpha$, IL-1b, IL-6, and IL-8 $(65,86)$ (Figure 1). Intrigu- 
ingly, IL-8 could be a survival factor for adipocytes in vivo, probably via stimulation of angiogenesis (89). Thus inflammatory cells in growing adipose tissues are coordinators for coupling the concomitant events of adipogenesis and angiogenesis.

TGF- $\beta$ and TF are increased in adipose tissue of obese mice, and both adipocytes and stromal cells express these factors (61). TGF- $\beta$ could positively and negatively regulate angiogenesis depending on the concentration and receptor types expressed in endothelial cells $(90,91)$. Preadipocytes and adipocytes produce high levels of HGF, which is an important angiogenic factor for vessel growth and remodeling $(60,92)$. Remarkably, Ang-2 as a vascular remodeling factor is consistently upregulated during adipose tissue growth $(62,92)$. These findings support the notion that adipose tissue development requires continuous remodeling, maturation, and patterning of the vasculature. Other angiogenic factors including VEGF-B, VEGF-C, and angiogenin have also been positively correlated with $\mathrm{BMI}(92,93)$.

Depending on dosages, PAI-1 can both positively and negatively regulate angiogenesis (94). When exposed to a high-fat diet, PAI-1-deficient mice have increased adipose tissue development accompanied by high vessel density, although conflicting results have also been reported (95-97). These paradoxical findings could be due to the composition and timing of diet, genetic background, and age of mice used (98). Taken together, these findings support the notion that adipose tissue development requires constant vascular remodeling and that multiple angiogenic molecules produced in adipose tissue may contribute to the complex regulation of adipogenesis (99).

\section{Adipose tissue-derived angiogenesis inhibitors}

Like most other adult tissues, the plasticity of adipose vasculature may represent the outcome of a net balance between angiogenic factors and inhibitors, which collectively determine growth or regression of the vasculature. Adipose tissue also produces several angiogenesis inhibitors. In contrast to proangiogenic factors, regulation of adipose vessel growth and remodeling by endogenous angiogenesis inhibitors is relatively poorly understood. As a specific adipocyte-derived hormone, adiponectin accumulates to high levels in the circulation of lean individuals and may protect against diabetes and atherosclerosis (100). Blood levels of adiponectin have inversely been correlated with BMI and are significantly decreased in obese animals and humans, suggesting its negative role in regulation of adipogenesis (101). Adiponectin inhibits endothelial cell proliferation, migration, and survival via activation of caspase-triggered endothelial cell apoptosis (102). In vivo it inhibits mouse corneal, CAM, and tumor angiogenesis. However, deletion or overexpression of adiponectin in mice does not seem to affect body weight, suggesting that the adiponectin system might be redundant (103).

Several reports describe the inexplicable finding that a number of endogenous angiogenesis inhibitors including endostatin, thrombospondin 1 (TSP-1), and soluble VEGFR-2 are produced at high levels in overweight and obese subjects $(62,92)$. In addition, an elevated level of PlGF has been found in expanding subcutaneous adipose tissues and PlGF might negatively regulate VEGF-induced angiogenesis by the formation of biologically inactive heterodimers $(62,104-107)$. Thus PlGF may indirectly inhibit adipose angiogenesis by negatively modulating angiogenic activity of VEGF. Why do growing adipose tissues produce high levels of angiogenesis inhibitors? Although there are no direct answers to this question, it is speculated that when the growth rate of an adipose tissue becomes stabilized, high expression levels of angiogenesis inhibitors are required to restrict further vessel growth. In agreement with this hypothesis, TSP-1 expression is downregulated in preadipocytes, followed by upregulation in differentiated adipocytes $(108,109)$. High expression of endostatin could be due to the fact that expanding adipose tissue produces an excessive amount of proteases such as MMPs that cleave collagen XVIII into endostatin (110). A common consistent finding from several reports is that Ang- 1 is remarkably downregulated in growing adipose tissues and plays a crucial role in vascular remodeling $(62,99$, 111). Decreased levels of Ang-1 would allow mural cells including pericytes and vascular smooth muscle cells to repel from blood vessels and to increase exposure and sensitivity of endothelial cells to other angiogenic factors such as VEGF. High levels of TGF- $\beta$ may also inhibit adipose tissue angiogenesis. Although TSP-1 is a relatively well characterized angiogenesis inhibitor, deletion of the TSP-1 gene in mice did not result in severe vasculature-related abnormalities (112). Similarly, exposure of TSP-1 knockout mice to a high-calorie diet does not significantly alter body weight or adipose tissue development as compared with control animals (113). Thus the role of TSP-1 in regulation of adipose angiogenesis warrants further investigation. Taken together from a balanced view of angiogenesis regulation, it is not surprising that a number of endogenous angiogenesis inhibitors are upregulated in order to maintain a homeostatic state of the adipose tissue by counteracting the excessive proangiogenic activity.

\section{Therapeutic intervention of obesity by antiangiogenic agents}

Concomitant occurrence of adipogenesis and angiogenesis has led us to believe that therapeutic intervention of obesity might be achieved by targeting the vasculature. Known angiogenesis inhibitors, including the small chemical compound TNP-470, and broad-spectrum endogenous protein inhibitors, including angiostatin and endostatin, have been tested in both genetically obese and high-calorie diet-fed obese animals $(16,18)$. Interestingly, administration of angiostatin, endostatin, and TNP-470 in both genetically obese and high-calorie diet-fed mice results in a dosedependent and reversible weight reduction as well as adipose tissue loss without significantly affecting food intakes $(16,18)$. Because angiogenesis inhibitors such as angiostatin and endostatin specifically target endothelial cells but not other cell types, the anti-obesity activity of these inhibitors is achieved via their antiangiogenic activity. Indeed, immunohistochemical analysis has revealed that vascularity of the adipose tissue is significantly reduced in animals treated with angiogenesis inhibitors (16). Furthermore, the angiogenesis inhibitor-treated adipose tissue undergoes remarkable vascular remodeling and contains an increased number of apoptotic cells and a decreased number of proliferating endothelial cells (18). Another interesting finding is that the angiogenesis inhibitor TNP-470 increases insulin sensitivity in obese animals, suggesting that these antiangiogenic agents might normalize insulin sensitivity and prevent the development of type II diabetes in addition to body weight gain (16).

\section{Future perspectives}

Both genetic and high-calorie diet-induced obesity requires the switch of an angiogenic phenotype in adipose tissues to support adipogenesis. Adipose tissue is unique in the body for its plastic- 
ity of growth and regression throughout life. This feature requires the coordinative growth and regression of the adipose vasculature. The crosstalk between adipocytes and endothelial cells is mediated by a number of angiogenic regulators, which cooperatively control vessel growth. It seems that adipose tissues located in different parts of the body express a different spectrum of angiogenic factors. For example, VEGF is a major angiogenic factor in omental fat, whereas it is not predominantly expressed in other adipose tissue. Consistently, obese animals are resistant to anti-VEGF therapy (18). Future studies on differential expression and function of various angiogenic factors in different adipose tissues would provide important information for designing therapeutic interventions.

Despite identification of multiple angiogenic factors in adipose tissue, little is known about the interplay between these factors in the adipose tissue environment. Coordinative communications between adipokines and other angiogenic factors are particularly important to understand the relationship of global control of adipose tissue expansion and local angiogenic responses. For example, high levels of serum leptin restrict fat mass expansion via endocrine stimulation of the central negative feedback loop, and paradoxically, leptin might promote adipogenesis through paracrine stimulation of angiogenesis. Inversely, high levels of serum adiponectin might restrain adipose tissue growth via inhibition of angiogenesis locally. Mechanistically, very little is known about how these adipokines are differentially regulated during adipose tissue growth. In addition to adiponectin, there are probably more endogenous angiogenesis inhibitors in the adipose to be discovered.

Can antiangiogenic agents be used for the treatment of existing obesity in humans? Although there is no definite answer to this question, there is a high probability that many of the angiogenic factors expressed in the adipose tissue might function as survival factors for the vasculature. For example, both VEGF and IGFs are important survival angiogenic factors for endothelial cells, and withdrawal of these factors may lead to endothelial cell apoptosis $(114,115)$. Thus it is possible that antiangiogenic agents might block the functions of these survival factors, leading to regression of preexisting adipose vasculature and shrinkage of the fat mass. This speculation warrants further investigation. At least in tumor studies, anti-VEGF agents have been reported to regress the established tumor vasculature and reduce tumor mass (115). Taken together, the data for antiangiogenic agents offer an exciting new therapeutic option for the prevention and treatment of obesity. As more antiangiogenic agents become available for experimental and clinical applications, they provide an outstanding opportunity to test this possibility in the near future.

\section{Acknowledgments}

The author thanks Yuan Xue for the artistic work and Anker Jon Hansen, Ebba Bråkenhielm, and Johan Nissen for critical reading of the manuscript. Research grants to the author's laboratory were received from the Swedish Research Council, the Swedish HeartLung Foundation, the Swedish Cancer Foundation, the Karolinska Institute Foundation, and the Torsten and Ragnar Söderberg's Foundations. The author is also supported by European Union Integrated Projects of Angiotargeting contract 504743 and VascuPlug contract STRP 013811.

Address correspondence to: Yihai Cao, Department of Microbiology, Tumor and Cell Biology, Karolinska Institute, SE-171 77 Stockholm, Sweden. Phone: 46-8-5248-7596, Fax: 46-8-33-13-999, E-mail: yihai.cao@ki.se.
1. Korner, J., and Aronne, L.J. 2003. The emerging science of body weight regulation and its impact on obesity treatment. J. Clin. Invest. 111:565-570. doi:10.1172/JCI200317953.

2. Friedman, J.M. 2000. Obesity in the new millennium. Nature. 404:632-634.

3. Kopelman, P.G. 2000. Obesity as a medical problem. Nature. 404:635-643.

4. Roth, J., Qiang, X., Marban, S.L., Redelt, H., and Lowell, B.C. 2004. The obesity pandemic: where have we been and where are we going? Obes. Res. 12(Suppl. 2):88S-101S.

5. Carmeliet, P. 2005. Angiogenesis in life, disease and medicine. Nature. 438:932-936.

6. Dvorak, H.F. 2005. Angiogenesis: update 2005. J. Thromb. Haemost. 3:1835-1842.

7. Ferrara, N., and Kerbel, R.S. 2005. Angiogenesis as a therapeutic target. Nature. 438:967-974.

8. Folkman, J. 1995. Angiogenesis in cancer, vascular, rheumatoid and other disease. Nat. Med. 1:27-31.

9. Folkman, J. 1995. Seminars in Medicine of the Beth Israel Hospital, Boston. Clinical applications of research on angiogenesis. N. Engl. J. Med. 333:1757-1763.

10. Lambert, P.D., et al. 2001. Ciliary neurotrophic factor activates leptin-like pathways and reduces body fat, without cachexia or rebound weight gain, even in leptin-resistant obesity. Proc. Natl. Acad. Sci.U.S. A. 98:4652-4657.

11. Lyden, D., et al. 2001. Impaired recruitment of bone-marrow-derived endothelial and hematopoietic precursor cells blocks tumor angiogenesis and growth. Nat. Med. 7:1194-1201.

12. McDonald, D.M., and Choyke, P.L. 2003. Imaging of angiogenesis: from microscope to clinic. Nat. Med. 9:713-725.

13. Bouloumie, A., Lolmede, K., Sengenes, C., Galitzky,
J., and Lafontan, M. 2002. Angiogenesis in adipose tissue. Ann. Endocrinol. (Paris). 63:91-95.

14. Crandall, D.L., Hausman, G.J., and Kral, J.G. 1997. A review of the microcirculation of adipose tissue: anatomic, metabolic, and angiogenic perspectives. Microcirculation. 4:211-232.

15. Silverman, K.J., et al. 1988. Angiogenic activity of adipose tissue. Biochem. Biophys. Res. Commun. 153:347-352.

16. Brakenhielm, E., et al. 2004. Angiogenesis inhibitor, TNP-470, prevents diet-induced and genetic obesity in mice. Circ. Res. 94:1579-1588.

17. Neels, J.G., Thinnes, T., and Loskutoff, D.J. 2004. Angiogenesis in an in vivo model of adipose tissue development. FASEB J. 18:983-985.

18. Rupnick, M.A., et al. 2002. Adipose tissue mass can be regulated through the vasculature. Proc. Natl. Acad. Sci. U. S. A. 99:10730-10735.

19. Lau, D.C. 1990. Nature and nurture in adipocyte development and growth. Int. J. Obes. 14(Suppl. 3): $153-157$.

20. Lau, D.C., Shillabeer, G., Wong, K.L., Tough, S.C., and Russell, J.C. 1990. Influence of paracrine factors on preadipocyte replication and differentiation. Int. J. Obes. 14(Suppl. 3):193-201.

21. Planat-Benard, V., et al. 2004. Plasticity of human adipose lineage cells toward endothelial cells: physiological and therapeutic perspectives. Circulation. 109:656-663.

22. Cao, Y., et al. 2005. Human adipose tissue-derived stem cells differentiate into endothelial cells in vitro and improve postnatal neovascularization in vivo. Biochem. Biophys. Res. Commun. 332:370-379.

23. Himms-Hagen, J., and Desautels, M. 1978. A mitochondrial defect in brown adipose tissue of the obese (ob/ob) mouse: reduced binding of purine nucleotides and a failure to respond to cold by an increase in binding. Biochem. Biophys. Res. Commun. 83:628-634.

24. Tonello, C., Dioni, L., Briscini, L., Nisoli, E., and Carruba, M.O. 1998. SR59230A blocks beta3adrenoceptor-linked modulation of upcoupling protein-1 and leptin in rat brown adipocytes. Eur. J. Pharmacol. 352:125-129.

25. Bukowiecki, L., et al. 1980. Mechanism of enhanced lipolysis in adipose tissue of exercise-trained rats. Am. J. Physiol. 239:E422-E429.

26. Cannon, B., Jacobsson, A., Rehnmark, S., and Nedergaard, J. 1996. Signal transduction in brown adipose tissue recruitment: noradrenaline and beyond. Int. J. Obes. Relat. Metab. Disord. 20(Suppl. 3):S36-S42.

27. Beck, C.S. 1935. Revascularization of the heart by vein graft from aorta to coronary sinus. Ann. Surg. 102:801-813.

28. Vineberg, A.M., et al. 1965. Myocardial revascularization by omental graft without pedicle: experimental background and report on 25 cases followed 6 to 16 months. J. Thorac. Cardiovasc. Surg. 49:103-129.

29. Castellot, J.J., Jr., Karnovsky, M.J., and Spiegelman, B.M. 1982. Differentiation-dependent stimulation of neovascularization and endothelial cell chemotaxis by 3T3 adipocytes. Proc. Natl. Acad. Sci. U. S. A. 79:5597-5601.

30. Goldsmith, H.S., Griffith, A.L., Kupferman, A., and Catsimpoolas, N. 1984. Lipid angiogenic factor from omentum. JAMA. 252:2034-2036.

31. Li, J., Yu, X., Pan, W., and Unger, R.H. 2002. Gene expression profile of rat adipose tissue at the onset of high-fat-diet obesity. Am. J. Physiol. Endocrinol. Metab. 282:E1334-E1341.

32. Zhang, Q.X., et al. 1997. Vascular endothelial growth factor is the major angiogenic factor in 
omentum: mechanism of the omentum-mediated angiogenesis. J. Surg. Res. 67:147-154.

33. Hutley, L.J., et al. 2001. Human adipose tissue endothelial cells promote preadipocyte proliferation. Am. J. Physiol. Endocrinol. Metab. 281:E1037-E1044.

34. Varzaneh, F.E., Shillabeer, G., Wong, K.L., and Lau, D.C. 1994. Extracellular matrix components secreted by microvascular endothelial cells stimulate preadipocyte differentiation in vitro. Metabolism. 43:906-912.

35. Hausman, G.J., and Richardson, L.R. 1982. Histochemical and ultrastructural analysis of developing adipocytes in the fetal pig. Acta Anat. (Basel). 114:228-247.

36. Crandall, D.L., Busler, D.E., McHendry-Rinde, B., Groeling, T.M., and Kral, J.G. 2000. Autocrine regulation of human preadipocyte migration by plasminogen activator inhibitor-1. J. Clin. Endocrinol. Metab. 85:2609-2614.

37. Fukumura, D., et al. 2003. Paracrine regulation of angiogenesis and adipocyte differentiation during in vivo adipogenesis. Circ. Res. 93:e88-e97.

38. Panigrahy, D., et al. 2002. PPAR $\gamma$ ligands inhibit primary tumor growth and metastasis by inhibiting angiogenesis. J. Clin. Invest. 110:923-932. doi:10.1172/JCI200215634

39. Ren, D., Collingwood, T.N., Rebar, E.J., Wolffe, A.P., and Camp, H.S. 2002. PPARgamma knockdown by engineered transcription factors: exogenous PPARgamma2 but not PPARgamma1 reactivates adipogenesis. Genes Dev. 16:27-32.

40. Rosen, E.D., et al. 1999. PPAR gamma is required for the differentiation of adipose tissue in vivo and in vitro. Mol. Cell. 4:611-617.

41. Xin, H., Geng, Y., Pramanik, R., and Choubey, D. 2003. Induction of p202, a modulator of apoptosis, during oncogenic transformation of NIH 3T3 cells by activated H-Ras (Q61L) contributes to cell survival. J. Cell. Biochem. 88:191-204.

42. Kawaguchi, N., et al. 2002. ADAM 12 protease induces adipogenesis in transgenic mice. Am.J. Pathol. 160:1895-1903.

43. Bouloumie, A., Sengenes, C., Portolan, G., Galitzky, J., and Lafontan, M. 2001. Adipocyte produces matrix metalloproteinases 2 and 9: involvement in adipose differentiation. Diabetes. 50:2080-2086.

44. Bergers, G., et al. 2000. Matrix metalloproteinase- 9 triggers the angiogenic switch during carcinogenesis. Nat. Cell Biol. 2:737-744.

45. Christiaens, V., and Lijnen, H.R. 2006. Role of the fibrinolytic and matrix metalloproteinase systems in development of adipose tissue. Arch. Physiol. Biochem. 112:254-259.

46. Maquoi, E., Demeulemeester, D., Voros, G., Collen, D., and Lijnen, H.R. 2003. Enhanced nutritionally induced adipose tissue development in mice with stromelysin-1 gene inactivation. Thromb. Haemost. 89:696-704.

47. Lijnen, H.R., Demeulemeester, D., Van Hoef, B., Collen, D., and Maquoi, E. 2003. Deficiency of tissue inhibitor of matrix metalloproteinase-1 (TIMP1) impairs nutritionally induced obesity in mice. Thromb. Haemost. 89:249-255.

48. Lijnen, H.R., et al. 2002. Matrix metalloproteinase inhibition impairs adipose tissue development in mice. Arterioscler. Thromb. Vasc. Biol. 22:374-379.

49. Fitzpatrick, T.E., and Graham, C.H. 1998. Stimulation of plasminogen activator inhibitor-1 expression in immortalized human trophoblast cells cultured under low levels of oxygen. Exp. Cell Res. 245:155-162.

50. Scannell, G., et al. 1995. Hypoxia-induced alterations of neutrophil membrane receptors. J. Surg. Res. 59:141-145.

51. Cho, C.H., et al. 2007. Angiogenic role of LYVE-1positive macrophages in adipose tissue. Circ. Res. 100:e47-e57.

52. Rehman, J., et al. 2004. Secretion of angiogenic and antiapoptotic factors by human adipose stromal cells. Circulation. 109:1292-1298.

53. Cao, Y. 2005. Opinion: emerging mechanisms of tumour lymphangiogenesis and lymphatic metastasis. Nat. Rev. Cancer. 5:735-743.

54. Baillargeon, J., and Rose, D.P. 2006. Obesity, adipokines, and prostate cancer (review). Int. J. Oncol. 28:737-745.

55. Friedman, J.M., and Halaas, J.L. 1998. Leptin and the regulation of body weight in mammals. Nature. 395:763-770.

56. Hiraoka, Y., et al. 2006. In situ regeneration of adipose tissue in rat fat pad by combining a collagen scaffold with gelatin microspheres containing basic fibroblast growth factor. Tissue Eng. 12:1475-1487.

57. Kuo, L.E., and Zukowska, Z. 2007. Stress, NPY and vascular remodeling: Implications for stress-related diseases. Peptides. 28:435-440.

58. Lijnen, H.R., et al. 2006. Impaired adipose tissue development in mice with inactivation of placental growth factor function. Diabetes. 55:2698-2704.

59. $\mathrm{Mu}, \mathrm{H}$., et al. 2006. Adipokine resistin promotes in vitro angiogenesis of human endothelial cells. Cardiovasc. Res. 70:146-157.

60. Saiki, A., Watanabe, F., Murano, T., Miyashita, Y., and Shirai, K. 2006. Hepatocyte growth factor secreted by cultured adipocytes promotes tube formation of vascular endothelial cells in vitro. Int. J. Obes. (Lond.) 30:1676-1684.

61. Samad, F., Pandey, M., and Loskutoff, D.J. 1998. Tissue factor gene expression in the adipose tissues of obese mice. Proc. Natl. Acad. Sci. U. S. A. 95:7591-7596.

62. Voros, G., et al. 2005. Modulation of angiogenesis during adipose tissue development in murine models of obesity. Endocrinology. 146:4545-4554.

63. Dobson, D.E., et al. 1990. 1-Butyryl-glycerol: a novel angiogenesis factor secreted by differentiating adipocytes. Cell. 61:223-230.

64. Wilkison, W.O., Choy, L., and Spiegelman, B.M. 1991. Biosynthetic regulation of monobutyrin, an adipocyte-secreted lipid with angiogenic activity. J. Biol. Chem. 266:16886-16891.

65. Wellen, K.E., and Hotamisligil, G.S. 2003. Obesity-induced inflammatory changes in adipose tissue. J. Clin. Invest. 112:1785-1788. doi:10.1172/ JCI200320514.

66. Wellen, K.E., and Hotamisligil, G.S. 2005. Inflammation, stress, and diabetes. J. Clin. Invest. 115:1111-1119. doi:10.1172/JCI200525102.

67. Sierra-Honigmann, M.R., et al. 1998. Biological action of leptin as an angiogenic factor. Science. 281:1683-1686.

68. Bouloumie, A., Drexler, H.C., Lafontan, M., and Busse, R. 1998. Leptin, the product of Ob gene, promotes angiogenesis. Circ. Res. 83:1059-1066.

69. Ring, B.D., et al. 2000. Systemically and topically administered leptin both accelerate wound healing in diabetic ob/ob mice. Endocrinology. 141:446-449.

70. Suganami, E., et al. 2004. Leptin stimulates ischemia-induced retinal neovascularization: possible role of vascular endothelial growth factor expressed in retinal endothelial cells. Diabetes. 53:2443-2448.

71. Ribatti, D., et al. 2001. Angiogenic activity of leptin in the chick embryo chorioallantoic membrane is in part mediated by endogenous fibroblast growth factor-2. Int. J. Mol. Med. 8:265-268.

72. Park, H.Y., et al. 2001. Potential role of leptin in angiogenesis: leptin induces endothelial cell proliferation and expression of matrix metalloproteinases in vivo and in vitro. Exp. Mol. Med. 33:95-102.

73. Cao, R., Brakenhielm, E., Wahlestedt, C., Thyberg, J., and Cao, Y. 2001. Leptin induces vascular permeability and synergistically stimulates angiogenesis with FGF-2 and VEGF. Proc. Natl. Acad. Sci. U. S. A. 98:6390-6395.

74. Grosfeld, A., et al. 2002. Hypoxia increases leptin expression in human PAZ6 adipose cells. Diabetolo- gia. 45:527-530

75. Makino, Y., et al. 2001. Inhibitory PAS domain protein is a negative regulator of hypoxia-inducible gene expression. Nature. 414:550-554.

76. Mandriota, S.J., et al. 2002. HIF activation identifies early lesions in VHL kidneys: evidence for sitespecific tumor suppressor function in the nephron. Cancer Cell. 1:459-468.

77. Tigno, X.T., Selaru, I.K., Angeloni, S.V., and Hansen, B.C. 2003. Is microvascular flow rate related to ghrelin, leptin and adiponectin levels? Clin. Hemorheol. Microcirc. 29:409-416.

78. Winters, B., et al. 2000. Reduction of obesity, as induced by leptin, reverses endothelial dysfunction in obese (Lep(ob)) mice. J. Appl. Physiol. 89:2382-2390.

79. Eriksson, A., et al. 2003. Small GTP-binding protein $\mathrm{Rac}$ is an essential mediator of vascular endothelial growth factor-induced endothelial fenestrations and vascular permeability. Circulation. 107:1532-1538.

80. Kamba, T., et al. 2006. VEGF-dependent plasticity of fenestrated capillaries in the normal adult microvasculature. Am. J. Physiol. Heart Circ. Physiol. 290:H560-H576.

81. Henson, M.C., and Castracane, V.D. 2000. Leptin in pregnancy. Biol. Reprod. 63:1219-1228.

82. Matsui, H., et al. 2007. Ischemia/reperfusion in rat heart induces leptin and leptin receptor gene expression. Life Sci. 80:672-680.

83. Ekstrand, A.J., et al. 2003. Deletion of neuropeptide Y (NPY) 2 receptor in mice results in blockage of NPY-induced angiogenesis and delayed wound healing. Proc. Natl. Acad. Sci. U. S. A. 100:6033-6038.

84. Lee, E.W., et al. 2003. Neuropeptide Y induces ischemic angiogenesis and restores function of ischemic skeletal muscles. J. Clin. Invest. 111:1853-1862. doi:10.1172/JCI200316929.

85. Movafagh, S., Hobson, J.P., Spiegel, S., Kleinman, H.K., and Zukowska, Z. 2006. Neuropeptide Y induces migration, proliferation, and tube formation of endothelial cells bimodally via Y1, Y2, and Y5 receptors. FASEB J. 20:1924-1926.

86. Coppack, S.W. 2001. Pro-inflammatory cytokines and adipose tissue. Proc. Nutr. Soc. 60:349-356.

87. Han, R.N., Post, M., Tanswell, A.K., and Lye, S.J. 2003. Insulin-like growth factor-I receptor-mediated vasculogenesis/angiogenesis in human lung development. Am. J. Respir. Cell Mol. Biol. 28:159-169.

88. Olszanecka-Glinianowicz, M., Zahorska-Markiewicz, B., Zurakowski, A., and Glinianowicz, M. 2005. The role of tumor necrosis factor (TNFalpha) in control of metabolism [In Polish]. Wiad. Lek. 58:670-674.

89. Shoshani, O., et al. 2005. The effect of interleukin-8 on the viability of injected adipose tissue in nude mice. Plast. Reconstr. Surg. 115:853-859.

90. Hotamisligil, G.S., Shargill, N.S., and Spiegelman, B.M. 1993. Adipose expression of tumor necrosis factor-alpha: direct role in obesity-linked insulin resistance. Science. 259:87-91.

91. Lebrin, F., Deckers, M., Bertolino, P., and Ten Dijke, P. 2005. TGF-beta receptor function in the endothelium. Cardiovasc. Res. 65:599-608.

92. Silha, J.V., Krsek, M., Sucharda, P., and Murphy, L.J. 2005. Angiogenic factors are elevated in overweight and obese individuals. Int. J. Obes. (Lond.) 29:1308-1314.

93. Asano, A., Irie, Y., and Saito, M. 2001. Isoform-specific regulation of vascular endothelial growth factor (VEGF) family mRNA expression in cultured mouse brown adipocytes. Mol. Cell. Endocrinol. 174:71-76.

94. Devy, L., et al. 2002. The pro- or antiangiogenic effect of plasminogen activator inhibitor 1 is dose dependent. FASEB J. 16:147-154.

95. Ma, L.J., et al. 2004. Prevention of obesity and insulin resistance in mice lacking plasminogen activator inhibitor 1. Diabetes. 53:336-346.

96. Morange, P.E., et al. 2000. Influence of PAI-1 on adipose tissue growth and metabolic parameters in 
a murine model of diet-induced obesity. Arterioscler. Thromb. Vasc. Biol. 20:1150-1154.

97. Schafer, K., Fujisawa, K., Konstantinides, S., and Loskutoff, D.J. 2001. Disruption of the plasminogen activator inhibitor 1 gene reduces the adiposity and improves the metabolic profile of genetically obese and diabetic ob/ob mice. FASEB J. 15:1840-1842.

98. Lijnen, H.R. 2005. Effect of plasminogen activator inhibitor-1 deficiency on nutritionally-induced obesity in mice. Thromb. Haemost. 93:816-819.

99. Dallabrida, S.M., et al. 2003. Adipose tissue growth and regression are regulated by angiopoietin-1. Biochem. Biophys. Res. Commun. 311:563-571.

100.Yamauchi, T., et al. 2001. The fat-derived hormone adiponectin reverses insulin resistance associated with both lipoatrophy and obesity. Nat. Med. 7:941-946.

101.Arita, Y., et al. 1999. Paradoxical decrease of an adipose-specific protein, adiponectin, in obesity. Biochem. Biophys. Res. Commun. 257:79-83.

102. Brakenhielm, E., et al. 2004. Adiponectin-induced antiangiogenesis and antitumor activity involve caspase-mediated endothelial cell apoptosis. Proc. Natl. Acad. Sci. U. S. A. 101:2476-2481.

103. Maeda, N., et al. 2002. Diet-induced insulin resis- tance in mice lacking adiponectin/ACRP30. Nat. Med. 8:731-737.

104.Eriksson, A., et al. 2002. Placenta growth factor-1 antagonizes VEGF-induced angiogenesis and tumor growth by the formation of functionally inactive PIGF-1/VEGF heterodimers. Cancer Cell. 1:99-108.

105.Inuzuka, H., et al. 1999. Differential regulation of immediate early gene expression in preadipocyte cells through multiple signaling pathways. Biochem. Biophys. Res. Commun. 265:664-668.

106. Seida, A., et al. 2003. Serum bFGF levels are reduced in Japanese overweight men and restored by a 6-month exercise education. Int.J. Obes. Relat. Metab. Disord. 27:1325-1331.

107.Shang, C.A., Thompson, B.J., Teasdale, R., Brown, R.J., and Waters, M.J. 2002. Genes induced by growth hormone in a model of adipogenic differentiation. Mol. Cell. Endocrinol. 189:213-219.

108.Burton, G.R., Nagarajan, R., Peterson, C.A., and McGehee, R.E., Jr. 2004. Microarray analysis of differentiation-specific gene expression during 3T3L1 adipogenesis. Gene. 329:167-185.

109. Okuno, M., et al. 2002. Isolation of up- or downregulated genes in PPARgamma-expressing NIH-
3T3 cells during differentiation into adipocytes. FEBS Lett. 519:108-112.

110. O'Reilly, M.S., et al. 1997. Endostatin: an endogenous inhibitor of angiogenesis and tumor growth. Cell. 88:277-285.

111.Yancopoulos, G.D., Klagsbrun, M., and Folkman, J. 1998. Vasculogenesis, angiogenesis, and growth factors: ephrins enter the fray at the border. Cell. 93:661-664.

112.Lawler, J., et al. 1998. Thrombospondin-1 is required for normal murine pulmonary homeostasis and its absence causes pneumonia. J. Clin. Invest. 101:982-992.

113.Voros, G., and Lijnen, H.R. 2006. Deficiency of thrombospondin- 1 in mice does not affect adipose tissue development. J. Thromb. Haemost. 4:277-278.

114. Mancuso, M.R., et al. 2006. Rapid vascular regrowth in tumors after reversal of VEGF inhibition. J. Clin. Invest. 116:2610-2621. doi:10.1172/JCI24612.

115.Polverino, A., et al. 2006. AMG 706, an oral, multikinase inhibitor that selectively targets vascular endothelial growth factor, platelet-derived growth factor, and kit receptors, potently inhibits angiogenesis and induces regression in tumor xenografts. Cancer Res. 66:8715-8721. 